\title{
Avaliação da extrusão apical de detritos, após a utilização das limas ProTaper Next e WaveOne, na instrumentação do sistema de canais radiculares
}

\section{Evaluation of the apical debris extrusion, after using ProTaper Nxt and WaveOne dental files, for root canal instrumentation}

\author{
Kizzy Esteves Ferreira ${ }^{1}$, Ludmila de Almeida Cunha ${ }^{1}$, Viviane Ferreira Guimarães Xavier ${ }^{1}$, \\ Mariane Floriano Lopes Santos Lacerda ${ }^{2}$, Caroline Felipe Magalhães Girelli ${ }^{1}$ \\ ${ }^{1}$ Curso de Especialização em Endodontia, Associação Brasileira de Odontologia Regional Governador \\ Valadares (ABO/GV) - Governador Valadares (MG), Brasil. \\ ${ }^{2}$ Departamento de Odontologia, Universidade Federal de Juiz de Fora (UFJF), Campus Governador Valadares \\ - Governador Valadares (MG), Brasil.
}

\begin{abstract}
Resumo
Apesar dos avanços tecnológicos no âmbito da instrumentação, extrusão de raspas de dentina, soluções de irrigação, medicação intracanal e polpa necrótica contendo micro-organismos, podem extruir pelo periápice, resultando em complicações pós-operatórias, como o flare up, com consequente retardo da cura periapical. O presente estudo comparou, por meio de uma revisão integrativa, a extrusão apical de detritos após a instrumentação utilizando as limas WaveOne e ProTaper Next. A seleção dos artigos foi realizada na base de dados Medical Literature and Retrieval System Online (PubMed), com a utilização dos seguintes descritores: Apical extrusion, WaveOne, ProTaper Next, Debris e Apically extruded debris. Como critério de inclusão, foram selecionados artigos de língua inglesa publicados durante o período de 2013 a 2017. Após realizada a leitura dos textos na íntegra, prosseguiu-se com a construção de uma tabela com informações levantadas e análise dos resultados. Todos os artigos selecionados foram publicados em periódicos de procedência internacional e da área odontológica. Verificou-se que os artigos foram publicados em anos diferentes, correspondendo ao total de um (20\%) em 2014, dois (40\%) em 2015 e dois (40\%) em 2016. Em relação aos periódicos, o Internacional Endodontic Journal publicou dois artigos, o Journal of Conservative Dentistry, dois artigos, e o European Journal of Dentistry, um artigo. Pode-se concluir que nenhum dos dois sistemas foi capaz de impedir a extrusão apical de detritos.
\end{abstract}

Palavras-chave: extrusão apical; endodontia; intrumentação.

\begin{abstract}
Despite the instrumentation technology advance, dentin scrapings extrusion, irrigation solutions, intracanal medication, sealer material and micro-organisms filled necrotic pulp, can extrude through the periapice, resulting in post operatory complications, like flare up, resulting in a periapical regeneration delay. The corresponding study compared, by a integrative review, the apical debris extrusion after instrumentation using 'Wave One' and 'ProTaper Next' dental files. The article selection was made on the database of Medical Literature and Retrieval System Online (PubMed), using the descriptors: Apical extrusion,
\end{abstract}

Autor para correspondência: Caroline Felipe Magalhães Girelli - Curso de Especialização em Endodontia, Associação Brasileira de Odontologia Regional Governador Valadares (ABO/GV) Av. Dr. Sérvulo Teixeira, 412 - Alto Esplanada - CEP: 35064-004 - Governador Valadares (MG), Brasil - E-mail: carolfmagalhaes3@hotmail.com

Recebido em: 25 de maio de 2017

Aceito para publicação em: 24 de setembro de 2017

http://dx.doi.org/10.4322/1980-0029.032017 
WaveOne, ProTaper Next, Debris, Apically extruded debris. Inclusion criteria consisted of, articles in English and published during the period of 2013 until 2017. After reading the articles, informational chart was created, containing the data analyses and information gathered. All the articles were published in relevant international newspapers, at the dentistry community. We verified that the articles were published in different years, corresponding to a total of $1(20 \%)$ at the year of 2014, $2(40 \%)$ at 2015 and $2(40 \%)$ at 2016. new identified 3 different journals, featuring the International Endodontic Journal, which published 2 articles, the Journal of Conservative Dentistry, which published 2 articles and the European Journal of Dentistry, which published 1 article. In conclusion, none of the systems were able to prevent the debris extrusion.

Keywords: apical extrusion; endodontics; intrumentation.

\section{Introdução}

A introdução da liga de níquel-titânio (Ni-Ti) na Endodontia trouxe grandes vantagens e facilidades durante a fase do preparo biomecânico do canal radicular (SOUZA et al., 2006), pois as limas produzidas a partir dessa liga apresentam maior flexibilidade do que as de aço inoxidável (GAMBARINI et al., 2008).

Instrumentos produzidos por um novo processo de tratamento térmico inovador, conhecido como M-wire, têm sido propostos para utilização na Endodontia. Essa nova liga aumenta a flexibilidade dos instrumentos, possibilitando preservar o curso anatômico original do canal, evitando degraus ao nível apical e transporte do forame, dando forma e contorno e, ao mesmo tempo, eliminando os restos pulpares e a quantidade de micro-organismos (SOUZA et al., 2006).

Recentemente, o sistema ProTaper Next foi introduzido no mercado para instrumentação dos canais radiculares. Essa técnica rotatória é constituída de três instrumentos para o preparo de corpo do canal, os denominados Shaping files. Apresentam maior variação do percentual da conicidade próximo ao intermediário e são constituídos pelos instrumentos $\mathrm{SX}, \mathrm{S} 1$ e S2 com D0, respectivamente, de $0,19 \mathrm{~mm}, 0,17 \mathrm{~mm}$ e $0,20 \mathrm{~mm}$. Os outros três instrumentos são denominados Finishing files e são instrumentos para o acabamento do preparo. Possuem maior conicidade próximo à guia e são constituídos pelos instrumentos F1 ( $\mathrm{D} 0=0,20 \mathrm{~mm}), \mathrm{F} 2(\mathrm{D} 0=0,25 \mathrm{~mm})$ e F3 (D0=0,30 mm) (DUARTE et al., 2009).

Outro sistema de instrumentação disponível no mercado, conhecido como WaveOne (Dentsply Maillefer, Ballaigues, VD, Switzerland), constitui um sistema reciprocante e tem uma conicidade reversa, um ângulo helicoidal variável e uma borda não ativa. É utilizado com rotação no sentido horário de $170^{\circ}$ (direção de corte) e rotação de $50^{\circ}$ no sentido anti-horário (BANE et al., 2015), o que diminui o estresse do instrumento endodôntico causado pela tensão e compressão que se produz no interior do canal radicular no momento da instrumentação. Além disso, favorece o preparo do canal radicular com somente um instrumento, de uso único: Small (diâmetro 0,21 e conicidade 0,06 ) para canais mais finos, Primary (diâmentro 0,25 e conicidade 0,08 ) para a maioria dos canais radiculares e Large (diâmetro 0,40 e conicidade 0,08 ) para os mais amplos (SILVA et al., 2012; VIVEKANANDHAN et al., 2016).

Durante a instrumentação dos sistemas de canais radiculares, independentemente do sistema utilizado, a extrusão de raspas de dentina e de polpa necrótica contendo micro-organismos pode causar complicações pós-operatórias, como o flare up, impedindo o sucesso da terapia endodôntica (UZUN et al., 2016). Ainda não há, no entanto, consenso na literatura sobre qual sistema oferece melhores resultados quanto à extrusão de detritos apical. Dessa forma, o objetivo do presente trabalho foi realizar uma revisão integrativa comparando a extrusão apical de detritos após a instrumentação utilizando as limas ProTaper Next e WaveOne.

\section{Material e Métodos}

Utilizou-se como método de pesquisa a revisão integrativa da literatura, por meio de busca bibliográfica na base de dados Medical Literature Analysis and Retrieval System Online (PubMed), com os seguintes descritores: Apical extrusion, WaveOne, ProTaper Next, Debris e Apically extruded debris. Foi feita uma seleção dos artigos que contemplavam de três a cinco palavras-chave. Para seleção das publicações a serem incluídas na revisão, os critérios de inclusão adotados foram: estudos primários, publicados no período de 2013 a 2017 no idioma inglês. Além disso, o conteúdo apresentado no resumo deveria estar concernente ao objetivo ora proposto, e apenas aqueles que haviam sido publicados 
na íntegra foram avaliados. Capítulos de livros, teses de doutorado, dissertações de mestrado e relatórios técnicos foram excluídos. A síntese final desenvolveu-se na forma descritiva, no que se refere aos objetivos, resultados e conclusões obtidos de cada um dos estudos. Tais dados foram agrupados por semelhança e organizados em categorias temáticas (Quadro 1).

\section{Resultados}

A revisão integrativa foi realizada após a conferência de resumos e dos critérios de elegibilidade preestabelecidos. Entre esses artigos pesquisados nas bases de dados, verificou-se o seguinte resultado: no PubMED, encontraram-se 20 artigos, dos quais, após a aplicação dos critérios de inclusão e exclusão, foram selecionados cinco, todos publicados em periódicos de procedência internacional e da área odontológica.

Verificou-se que os artigos foram publicados em anos diferentes, correspondendo ao total de um (20\%) em 2014, dois (40\%) em 2015 e dois (40\%) em 2016. Em relação aos periódicos, o Internacional Endodontic Journal publicou dois artigos, o Jornal of Conservative Dentistry, dois artigos, e o European Journal of Dentistry, um artigo.

\section{Discussão}

Uma das principais causas do insucesso endodôntico é atribuída à extrusão de detritos infectados do canal radicular para os tecidos perirradiculares, provocando dor pós-operatória (EHSANI et al., 2016). No entanto, apesar dos

Quadro 1. Identificação dos estudos selecionados para a revisão integrativa.

\begin{tabular}{|c|c|c|c|}
\hline Artigo & Autores/Ano & Metodologia & Conclusão \\
\hline $\begin{array}{l}\text { Comparison of apically } \\
\text { extruded debris after large } \\
\text { apical preparations by } \\
\text { full-sequence rotary and } \\
\text { single-file reciprocating } \\
\text { systems. }\end{array}$ & Silva et al. (2016) & $\begin{array}{l}\text { Materiais extruídos } \\
\text { apicalmente foram } \\
\text { recolhidos em vidros } \\
\text { pré-pesados. O peso } \\
\text { médio de detritos foi } \\
\text { avaliado utilizando uma } \\
\text { microbalança. }\end{array}$ & $\begin{array}{l}\text { Todos os sistemas causaram } \\
\text { a extrusão de detritos. } \\
\text { Os sistemas ProTaper Next } \\
\text { e WaveOne extruíram de } \\
\text { forma semelhante. }\end{array}$ \\
\hline $\begin{array}{l}\text { Evaluation of apically } \\
\text { extruded debris associated } \\
\text { with several Ni-Ti systems }\end{array}$ & Üstün et al. (2014) & $\begin{array}{l}\text { Os detritos extruídos } \\
\text { apicalmente foram } \\
\text { recolhidos e depositados em } \\
\text { tubos Eppendorf e depois } \\
\text { foram pesados. }\end{array}$ & $\begin{array}{l}\text { Todos as técnicas } \\
\text { apresentaram extrusão } \\
\text { apical de detritos. } \\
\text { No sistema WaveOne, } \\
\text { houve uma menor extrusão } \\
\text { de detritos. }\end{array}$ \\
\hline $\begin{array}{l}\text { Quantitative evaluation } \\
\text { of apically extruded } \\
\text { debris during root canal } \\
\text { instrumentation with } \\
\text { ProTaper Universal, } \\
\text { ProTaper Next,WaveOne, } \\
\text { and self-adjusting file } \\
\text { systems. }\end{array}$ & Ozsu et al. (2014) & $\begin{array}{l}\text { Os detritos foram coletados } \\
\text { em tubos de Eppendorf e } \\
\text { depois pesados. }\end{array}$ & $\begin{array}{l}\text { Não houve diferença entre } \\
\text { os sistemas ProTaper Next e } \\
\text { WaveOne. }\end{array}$ \\
\hline $\begin{array}{l}\text { The self-adjusting file } \\
\text { instrumentation results } \\
\text { in less debris extrusion } \\
\text { apically when compared } \\
\text { to WaveOne and ProTaper } \\
\text { Next. }\end{array}$ & Pawar et al. (2015) & $\begin{array}{l}\text { Foram feitas as coletas dos } \\
\text { detritos apicais em tubos } \\
\text { Eppendorf e então pesados. }\end{array}$ & $\begin{array}{l}\text { Em todos os sistemas, } \\
\text { houve extrusão de detritos. } \\
\text { ProTaper Next extruiu } \\
\text { menos detritos que } \\
\text { WaveOne. }\end{array}$ \\
\hline $\begin{array}{l}\text { Comparison of apically } \\
\text { extruded debris associated } \\
\text { with several nickel- } \\
\text { titanium systems after } \\
\text { determining working } \\
\text { length by apex locator }\end{array}$ & Çiçek et al. (2016) & $\begin{array}{l}\text { Os detritos apicais foram } \\
\text { armazenados em tubos de } \\
\text { Eppendorf e depois pesados. }\end{array}$ & $\begin{array}{l}\text { Todos promoveram } \\
\text { extrusão, mas WO extruiu } \\
\text { menos que os demais } \\
\text { sistemas. }\end{array}$ \\
\hline
\end{tabular}


diversos instrumentos disponíveis no mercado, não se sabe qual o sistema que causa menor extrusão de detritos apicalmente.

Todas as instrumentações, independentemente da técnica utilizada, devem adotar medidas preventivas, evitando os problemas pós-operatórios. O flare-up tem sido relatado como o principal problema após o preparo químico mecânico a ser evitado e tem sido apontado como decorrente da irritação química, extrusão apical de irrigantes ou detritos (AKBAR, 2015; JAYAKODI et al., 2012).

Esta revisão integrativa teve como intuito avaliar a extrusão de detritos usando as Limas WaveOne e ProTaper Next. Após análise dos resultados, pôde-se verificar que a WaveOne, em $40 \%$ dos artigos, apresentou melhores resultados, enquanto que a ProTaper Next obteve maior eficácia em $20 \%$ dos trabalhos. A ausência de diferença entre os instrumentos avaliados esteve presente em $40 \%$ dos casos.

AProTaper Next foi selecionada para este trabalho por ter uma maior flexibilidade e resistência, reduzindo o risco de fratura do instrumento, apresentando conicidades variadas no mesmo instrumento, permitindo uma melhor limpeza, mantendo sua alta eficiência no corte e preservando a anatomia original do canal radicular, além de permitir uma instrumentação de canais mais complexos, atrésicos e com curvaturas mais acentuadas (VYVER; SCIANAMBLO, 2013).

Já a WaveOne foi selecionada por ter limas mais flexíveis e resistentes à fadiga e por possuir uma seção que varia ao longo eixo do instrumento, com conicidades variadas nos diâmetros apicais e na parte média da lima, além de apresentar um formato de triângulo com lados convexos na região mais próxima à ponta do instrumento, promovendo uma melhor limpeza e diminuindo significativamente o tempo de trabalho (SILVA et al., 2012).

O sistema reciprocante apresentou aumento do acúmulo de detritos. Isso ocorreu porque o movimento extrui detritos apicalmente, provocando maior acúmulo no ápice (BÜRKLEIN; SCHÄFER, 2012). O sistema rotatório proporciona corte mais aprimorado, e os detritos são carregados para fora do canal, evitando o acúmulo apical (UZUNOGLU et al., 2015). Dessa forma, ProTaper Next foi considerada como um sistema que provoca pouca extrusão apical de detritos durante a instrumentação, pois apresenta um design único com um centro de compensação de massa e de rotação. Com esse design, as chances de entupimento do sistema de canal radicular são poucas (PAWAR et al., 2015).

Contrariamente aos demais estudos, De-Deus et al. (2010) relataram que a instrumentação do canal radicular com apenas uma lima, em vez de sistemas com várias limas, diminui o número de paredes tocadas durante a instrumentação, uma das razões pelas quais os sistemas reciprocantes resultam menos extrusão de detritos. Corroborando esse achado, no presente estudo, o sistema WaveOne apresentou uma menor quantidade de raspas de dentina e de irrigantes extruídos apicalmente, quando comparado à ProTaper Next (USTUN et al., 2014).

Essa diferença deve-se ao fato não só do numero de limas utilizadas no sistema reciprocante, mas também porque esse sistema trabalha em grande rotação no sentido de corte (sentido horário) e em movimento alternativo no menor ângulo de rotação na direção de desgaste (sentido anti-horario), impedindo que os detritos acumulem na porção apical do canal e, consequentemente, extruindo para região periapical (ÇIÇEK et al., 2016).

Em contrapartida, no presente trabalho, alguns estudos não encontraram diferença na instrumentação entre os dois sistemas, WaveOne e ProTaper Next, pois ocorreu extrusão de detritos em ambos os sistemas, afirmando que nenhum sistema é totalmente isento de acúmulo de raspa de dentinas no ápice. A esse resultado atribui-se a largura apical de cada dente, ou seja, o fator que irá definir se houve mais ou menos extrusão apical é o diâmetro apical do preparo (OZSU et al., 2014; SILVA et al., 2016).

Dessa forma, fatores que afetam a quantidade de extrusão apical e de dor pós-operatória incluem: design, quantidade de limas, tamanho do instrumento, diâmetro de patência e instrumentação apical (VIVEKANANDHAN et al., 2016).

\section{Conclusão}

Com base nos artigos desta revisão integrativa sobre extrusão apical de detritos usando as limas ProTaper Next e WaveOne, concluiu-se que nenhum dos dois sistemas foi capaz de impedir a extrusão apical de detritos.

\section{Referências}

AKBAR, I. Efficacy of prophylactic use of antibiotics to avoid flare up during root canal treatment of nonvital teeth: a randomized clinical trial. Journal of Clinical and Diagnostic Research, v. 9, n. 3, p. ZC08-ZC11, 2015. PMid:25954695. 
BANE, K. et al. Root canal shaping by singlefile systems and rotary instruments: a laboratory study. Iranian Endodontic Journal, v. 10, n. 2, p. 135-139, 2015. PMid:25834600.

BÜRKLEIN, S.; SCHÄFER, E. Apically extruded debris with reciprocating single-file and full-sequence rotary instrumentation systems. Journal of Endodontics, v. 38, n. 6, p. 850-852, 2012. PMid:22595125. http://dx.doi.org/10.1016/j. joen.2012.02.017.

ÇIÇEK, E.; AKKOCAN, O.; FURUNCUOGLU, F. Comparison of apically extruded debris associated with several nickel-titanium systems after determining working length by apex locator. Journal of Conservative Dentistry, v. 19, n. 1, p. 68-71, 2016. PMid:26957797. http://dx.doi. org/10.4103/0972-0707.173203.

DE-DEUS, G. et al. Assessment of apically extruded debris produced by the single-file ProTaper F2 technique under reciprocating movement. Oral Surgery, Oral Medicine, Oral Pathology, Oral Radiology, and Endodontics, v. 110, n. 3, p. 390-394, 2010. PMid:20727500. http://dx.doi. org/10.1016/j.tripleo.2010.04.020.

DUARTE, M. A. H. et al. Sistema Pro Taper: análise técnica e científica. Salusvita, v. 28, n. 2 , p. 157-168, 2009.

EHSANI, M. et al. Comparison of apical extrusion of debris by using single-file, full-sequence rotary and reciprocating systems. Journal of Dentistry, v. 13 , n. 6, p. 394-399, 2016. PMid:28243300.

GAMBARINI, G. et al. Fatigue resistance of engine-driven rotary nickel-titanium instruments produced by new manufacturing methods. Journal of Endodontics, v. 34, n. 8, p. 1003-1005, 2008. PMid:18634935. http://dx.doi.org/10.1016/j. joen.2008.05.007.

JAYAKODI, H. et al. Clinical and pharmacological management of endodontic flare-up. Journal of Pharmacy \& Bioallied Sciences, v. 4, n. 2, p. S294-S298, 2012.

OZSU, D. et al. Quantitative evaluation of apically extruded debris during root canal instrumentation with ProTaper Universal, ProTaper Next, WaveOne, and self-adjusting file systems. European Journal of Dentistry, v. 8, n. 4, p. 504-508, 2014.
PAWAR, A. M. et al. The self-adjusting file instrumentation results in less debris extrusion apically when compared to WaveOne and ProTaper Next. Journal of Conservative Dentistry, v. 18, n. 2, p. 89-93, 2015.

SILVA, E. J. N. L. et al. Comparison of apically extruded debris after large apical preparations by full $\square$ sequence rotary and single $\square$ file reciprocating systems. International Endodontic Journal, v. 49, n. 7, p. 700-705, 2016. PMid:26174577. http:// dx.doi.org/10.1111/iej.12503.

SILVA, P. A. A. et al. Comparison of hardtissue debris produced by waveone and protaper instruments on mesial roots of mandibular molars. Revista Odontológica do Brasil Central, v. 21, n. 56, p. 560-563, 2012.

SOUZA, R. E.; BERNARDINELI, N.; BRAMANTE, C. M. Evaluation of the efficacy of instrumentation methods and chemical substances on the root canal walls: scanning electron microscopy study. Revista Odonto Ciência, v. 21, n. 52, p. 125-131, 2006.

USTUN, Y. et al. Evaluation of apically extruded debris associated with several Ni-Ti systems. International Endodontic Journal, v. 48, n. 7, p. 701-704, 2014. PMid:25112960.

UZUN, I. et al. Apical extrusion of debris using reciprocating files and rotary instrumentation systems. J Clin Practice, v. 19, n. 1, p. 71-75, 2016. PMid:26755222.

UZUNOGLU, E.; TURKER, S. A.; GÖRDUYSUS, M. Effects of different rotary files combined with different irrigation needles on apically extruded debris. Brazilian Dental Journal, v. 26, n. 4, p. 347-350, 2015. PMid:26312970. http://dx.doi. org/10.1590/0103-6440201300166.

VIVEKANANDHAN, P. et al. Comparison of apical debris extrusion of two rotary systems and one reciprocating system. Journal of Conservative Dentistry, v. 19, n. 3, p. 245-249, 2016. PMid:27217638. http://dx.doi.org/10.4103/09720707.181941 .

VYVER, P. J. V.; SCIANAMBLO, M. J. Clinical guidelines for the use of ProTaper Next instruments: part one. Endodontic Practice, v. 16, n. 4, p. 33-40, 2013. 